\title{
Retrospective versus prospective processes in delayed matching to sample
}

\author{
THOMAS B. STONEBRAKER and MARK RILLING \\ Michigan State University, East Lansing, Michigan
}

\begin{abstract}
Current views of delayed matching-to-sample (DMTS) performance in pigeons postulate the active maintenance (rehearsal) of a representation of the sample stimulus during the delay interval. Honig and Wasserman (1981) termed this type of processing retrospective. In an alternative account, prospective processing, a representation of an upcoming comparison (test) stimulus is maintained during the delay interval, rather than a representation of the sample. The present experiments attempted to determine whether processing is retrospective or prospective in DMTS, as well as in delayed simple discrimination (a related procedure). The results of the experiments indicated that although pigeons are capable of retrospective processing, prospective processing appears to be the process typically used when there are no constraints that would hinder or prevent its use. This finding is contrary to the usual assumption of retrospective processing.
\end{abstract}

Short-term or active memory in laboratory animals has been a popular topic in recent years, with the development of memory tasks such as delayed matching to sample (DMTS). Much of the recent research investigating how animals are able to perform tasks such as DMTS has favored the interpretation of an active process (rehearsal) of some coded form of an initial sample stimulus (which is no longer present) to bridge the delay between sample and comparison stimuli (Grant, 1981; Maki, 1981; Maki \& Hegvik, 1980; Maki, Olson, \& Rego, 1981; Roitblat, 1980; Stonebraker \& Rilling, 1981; Stonebraker, Rilling, \& Kendrick, 1981; Wagner, 1976). It is often assumed that the code refers to visual attributes of the sample stimulus (Maki, Riley, \& Leith, 1976; Roberts, 1972; Roberts \& Grant, 1976; Ruggiero \& Flagg, 1976) or some other mnemonic copy of the sample. This position assumes that animals perform DMTS by actively rehearsing the sample (maintaining some visual attribute of the sample stimulus) throughout the retention interval between sample and comparison stimuli. This sample rehearsal has been termed retrospective processing by Honig and Wasserman (1981).

Although the conventional interpretation has been that animals actively process the sample during the retention interval, it is equally possible that animals actively process or anticipate the upcoming comparison stimulus in order to perform accurately in DMTS (Roitblat, 1980). This comparison rehearsal was

This research was supported in part by Grant BNS-81-1096. from the National Science Foundation to Mark Rilling at Michigan State University. Reprints may be obtained from Thomas B. Stonebraker, Department of Psychology, Greenville College, Greenville, Illinois 62246. termed prospective processing by Honig and Wasserman. (For a complete discussion of the distinction between prospection and retrospection, see Wasserman, in press.) Correct performance on a retention test is not sufficient to determine what the properties of the memory trace are (whether processing is prospective or retrospective). Riley, Cook, and Lamb (1981) pointed out that the fact that an animal can match to sample after a delay interval shows that the animal is capable of bridging the gap between the initial event and the choice situation, nothing more. Accurate matching gives no indication of whether the process being used to span the retention interval is prospective versus retrospective.

If processing during the retention interval is retrospective, the following events would take place on a single DMTS trial: Following sample termination, some representation of the sample would be maintained throughout the retention interval. Upon stimuli presentation, a decision rule would be retrieved from long-term memory as the stimulus(i) is (are) compared with the representation of the sample stimulus currently in memory. The decision rule would indicate which stimulus is correctly paired with the sample (i.e., which stimulus is a match). However, if animals process a comparison stimulus during the retention interval, the sequence of events would be somewhat different. According to this prospective account, a sample stimulus would retrieve a representation of the comparison stimulus while the sample stimulus was still present. The organism would then code an instruction to respond to a specific comparison stimulus, and a representation of that stimulus would be maintained throughout the retention interval in an anticipatory fashion. Upon presentation of comparison stimuli, the organism would perform ac- 
curately by responding to the comparison that was in some way isomorphic to the representation currently being maintained in active memory. Good DMTS performance is equally possible with either of the above strategies. The question of interest in this series of experiments is whether pigeons retrospectively or prospectively process during the retention interval in DMTS.

The following experiments were designed to examine whether retention-interval processing is prospective or retrospective in DMTS. This was accomplished by ensuring that the possible codes for the sample and comparison stimuli had discriminably different properties (sample and comparisons that were not isomorphic). In addition, the training situation was arranged such that the tasks could be performed either retrospectively or prospectively.

\section{EXPERIMENT 1}

This experiment attempted to determine whether processing during DMTS is prospective or retrospective in nature by training birds with cues that perfectly predicted comparison sets (either identity matching, with red and green comparison stimuli, or symbolic matching, with vertical and horizontal lines as comparison stimuli) and occasionally testing birds with incorrect comparison sets. The logic behind the use of instructional stimuli was to provide a task that could be performed using either prospective or retrospective processing.

\section{Method}

Subjects. Four experimentally naive adult White Carneaux pigeons were used. The birds were maintained as in Experiment 1. of their free-feeding weights. The birds were housed individually in a temperature-controlled and constantly illuminated room and had free access to water and grit.

Apparatus. A standard Lehigh Valley Electronics three-key conditioning chamber was used. Interior dimensions were $35 \times 35$ $x 30 \mathrm{~cm}$. The $2.5-\mathrm{cm}$ response key could be transilluminated with red $(606 \mathrm{~nm})$, green $(555 \mathrm{~nm})$, a 1.0 -cm-diam circle, a $1.0-\mathrm{cm}$ equilateral triangle, or a vertical or horizontal $0.3 \times 2.5 \mathrm{~cm}$ white line on a black background. The stimuli were produced by an Industrial Electronics projector (Model 10-3723-757-L). Activation of a Lehigh Valley Electronics photoelectronic relay initiated the reinforcement timer. An exhaust fan, located on the wall opposite the response panel, partially masked extraneous noises. Experimental events were controlled by standard electromechanical programming equipment located in an adjacent room, with a paper-tape reader controlling the sequence of events.

Procedure. All birds were magazine trained and autoshaped according to the procedure described by Stonebraker and Rilling (1981). Following autoshaping, the birds were trained on a twochoice DMTS task. During DMTS training, a sample stimulus was presented (either red or green) and was terminated with the first keypeck after $12 \mathrm{sec}$ had elapsed. Red and green samples were pseudorandomly presented such that the same color did not appear more than three times in succession and each occurred equally often per session. Superimposed on the sample stimulus was either a circle or a triangle, which continued on the key throughout the retention interval, which followed the sample. Triangles predicted the occurrence of line-orientation (vertical and horizontal) comparison stimuli (symbolic matching), and circles predicted the occurrence of wavelength (red and green) compari- son stimuli (identity matching). These stimuli terminated at the end of the retention interval, prior to the onset of comparison stimuli. The four comparison stimuli, either vertical and horizontal lines or red and green, were presented simultaneously on side keys. Comparison stimuli were counterbalanced such that each color and line occurred an equal number of times on each side key. A single keypeck to the comparison stimulus that matched the sample terminated both comparison stimuli and resulted in reinforcement. A peck to the nonmatching comparison terminated both stimuli and initiated a new trial. The intertrial interval was $30 \mathrm{sec}$.

Throughout Experiment 1, reinforcement consisted of 2.0-sec access to mixed grain. Seventy-two trials were given each session, 36 identity and 36 symbolic matching. All trial types and combinations of stimuli occurred an equal number of times.

From the responses to matching and nonmatching comparison stimuli, discrimination ratios were calculated as a measure of matching accuracy. These ratios were calculated by dividing the responses during all matching comparison stimuli by the total number of responses during all comparison stimuli, both matching and nonmatching, and multiplying by 100 . A discrimination ratio of $100 \%$ indicates perfect matching performance, with responding during comparison stimuli occurring exclusively on matching trials. A ratio of $50 \%$ indicates chance levels of matching performance, with equal levels of responding occurring on matching and nonmatching trials.

During training, the retention interval was initially set at $1 \mathrm{sec}$. This interval was maintained until a bird performed above an $80 \%$ discrimination ratio on both matching tasks for two consecutive sessions. The retention interval was increased in 1-sec increments each time the criterion was met, until the terminal value of $4 \mathrm{sec}$ was reached.

Probe sessions began when a bird performed at or above $80 \%$ on both tasks for four consecutive sessions. Probe sessions were given every other session, or less frequently, as was needed to maintain baseline matching performance above $80 \%$. Probe sessions consisted of 64 standard trials and 8 probe trials. On probe trials, triangles were now paired with wavelength comparison stimuli (identity matching), and circles were now paired with lineorientation comparison stimuli (symbolic matching). Comparisonstimuli locations were counterbalanced, and all trial types were probed during each session. The 8 probe trials were therefore: red (sample) + circle:vertical (left)/horizontal (right); red + circle: horizontal/vertical; red + triangle:red/green; red + triangle:green/ red; green + circle:vertical/horizontal; and so on. Reinforcement contingencies were identical to those on standard trials. Fifteen probe sessions were given to each bird.

\section{Results}

Discrimination ratios were calculated from the choice responses to comparison stimuli, both for standard and for probe trials. The results for the seven birds that were tested are presented in Figure 1. Matching performance of $100 \%$ indicates perfect matching, whereas performance at $50 \%$ indicates chance (equal responding to matching and nonmatching comparison stimuli). For all birds, there was a decrement in matching performance on probe trials in relation to standard (baseline) trials. This decrement existed whether the task was identity or symbolic matching. The average matching percentage was $89.5 \%$ on baseline trials and $72.2 \%$ on probe trials.

\section{EXPERIMENT 2}

Experiment 2 also attempted to determine whether 

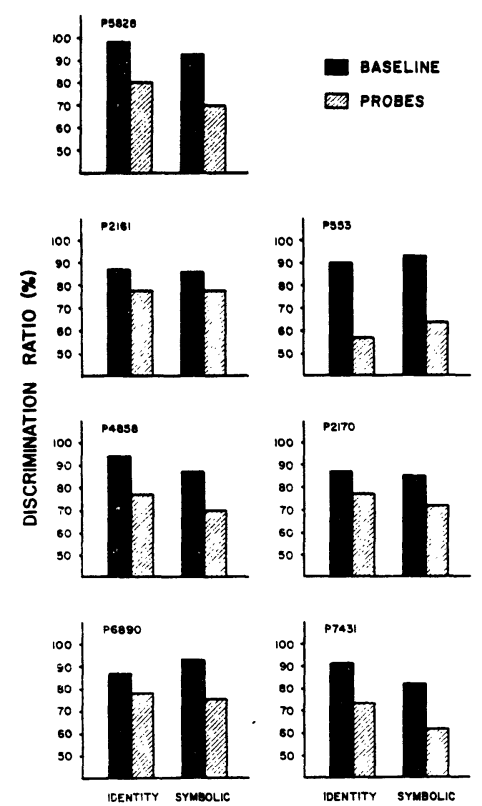

MATCHING TASK

Figure 1. Matching performance on baseline and probe trials for each task. Baseline trials were trials in which cues correctly predicted the matching task. Probe trials were trials in which the matching task was incorrectly cued. A discrimination ratio of $100 \%$ indicates perfect performance, with responding to comparison stimuli occurring exclusively on matching trials. A discrimination ratio of $50 \%$ indicates chance performance, with equal response levels to matching and nonmatching comparison stimuli.

the processing utilized by pigeons in short-term memory tasks is prospective or retrospective in nature. In Experiment 1, both delayed matching tasks were conditional; that is, the correct discriminative response at the end of the trial depended upon both the sample and the comparison stimuli, and changed from one trial to another. Honig and Wasserman (1981) described a delayed simple discrimination in which the sample stimuli signaled different outcome contingencies in the presence of a single test stimulus. Memory on these tasks was typically more robust than memory during delayed matching trials. In Experiment 2 , the birds were simultaneously trained on one delayed matching task and one delayed simple discrimination.

\section{Method}

Subjects. Eleven experimentally naive adult White Carneaux pigeons were used. The birds were maintained as in Experiment 1.

Apparatus. The apparatus was similar to that employed in Experiment 1.

Procedure. All birds were magazine trained and autoshaped according to the procedure described in Stonebraker and Rilling (1981). Following autoshaping, the birds were simultaneously trained on a successive DMTS and a delayed simple discrimination. For both tasks, a sample stimulus was presented (either red or green) and was terminated with the first keypeck after $12 \mathrm{sec}$ had elapsed. If no keypeck was emitted during the 30-sec period following the end of the 12-sec interval, sample stimuli automatically terminated. Superimposed on the sample stimulus was either a vertical or a horizontal line, which continued on the key throughout the retention interval (which followed the sample). Vertical lines predicted the occurrence of wavelength comparison stimuli (delayed matching task), and horizontal lines predicted a white comparison stimulus (delayed simple discrimination). Comparison stimuli were presented for a minimum of $5 \mathrm{sec}$. A $40-\mathrm{msec} 1000-\mathrm{Hz}$ tone of approximately $80 \mathrm{~dB}$ was presented at the onset of each comparison stimulus as an additional cue indicating the presence of that stimulus. In the DMTS task, comparison stimuli terminated automatically after $5 \mathrm{sec}$ on nonmatching trials (red-green and green-red) and were terminated with a single keypeck after $5 \mathrm{sec}$ on matching trials (red-red and greengreen). This keypeck also resulted in reinforcement. In the delayed simple discrimination, the white comparison terminated automatically after $5 \mathrm{sec}$ following green sample stimuli (S-) and were terminated with a single keypeck after $5 \mathrm{sec}$ following red sample stimuli $(\mathbf{S}+)$, and this keypeck resulted in reinforcement. The intertrial interval was $30 \mathrm{sec}$.

Throughout Experiment 2, reinforcement consisted of 2.5-sec access to mixed grain. All trial types occurred equally often and were presented pseudorandomly, as in Experiment 1. Since there were four trial types in the DMTS task (red-red, red-green, greenred, and green-green) and only two in the delayed simple discrimination (red-white and green-white), 48 of the 72 trials per session were DMTS and the remaining 24 were delayed simple discrimination.

During training, the retention interval was initially set at $1 \mathrm{sec}$. The retention interval was increased in 1 -sec increments each time the $80 \%$ discrimination criterion was met (as in Experiment 1) until a 3-sec retention interval was reached.

Probe sessions began when a bird performed at or above $80 \%$ on both tasks for four consecutive sessions, and were given every other session or less frequently, as in Experiment 1. Probe sessions consisted of 66 standard and 6 probe trials, one of each trial type. Contrary to training, on probe trials vertical line cues were followed by a white comparison and horizontal line cues were followed by either a red or a green comparison stimulus. Reinforcement contingencies on probe trials were identical to those on standard trials. A total of five probe sessions were given to each bird.

\section{Results}

Discrimination ratios were calculated from the response rates to comparison stimuli, both for baseline and for probe trials. These ratios are presented in Figure 2. For all birds, there was a decrement in matching performance on probe trials in relation to standard (baseline) trials. This decrement existed whether the task was DMTS or delayed simple discrimination. The average matching percentage was $83.5 \%$ on baseline trials and $51.8 \%$ on probe trials.

\section{DISCUSSION}

Roitblat (1980) presented data that he believed suggested that pigeon DMTS performance utilizes the processing of a representation isomorphic with the test (comparison) stimulus-prospective processing. The results of both experiments in the present study support the conclusion drawn by Roitblat that prospective processing can occur in delayed matching. These findings are contrary to the common assumption that processing involves a representation isomorphic with the sample stimulus (retrospective processing).

In retrospective processing, birds would process a representation of the sample stimulus on a particular trial. This process would be independent of the upcoming comparison stimuli, making the superimposed cues in the present studies irrelevant. Changing the meaning of the cues on probe trials should not influence matching performance if retrospective processing is used. However, the 

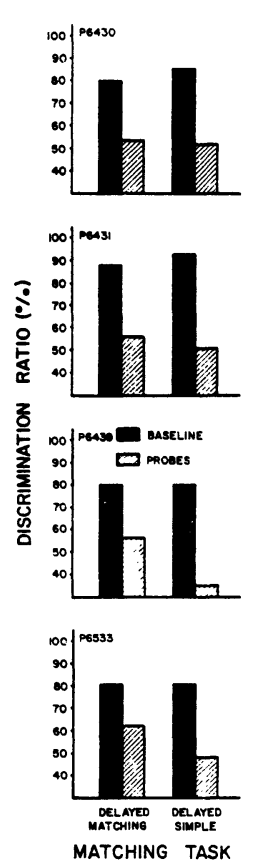

Figure 2. Matching performance on baseline and probe trials for each task. Baseline trials were trials in which cues correctly predicted the matching task. Probe trials were trials in which the matching task was incorrectly cued. A discrimination ratio of $100 \%$ indicates perfect performance, with responding to comparison stimuli occurring exlusively on matching trials. A discrimination ratio of $50 \%$ indicates chance performance, with equal response levels to matching and nonmatching comparison stimuli.

results of the present experiments indicate that the instructional cues do gain control over processing. Incorrectly cuing the type of matching task consistently resulted in a decrement in performance, when compared with performance on correctly cued trials. Although these results are inconsistent with a retrospective-process position, they are precisely what would be predicted with a prospective-process position. In prospective processing, birds would process a representation of one of the comparison stimuli on a particular trial, either the correct comparison (to approach) or the incorrect comparison (to withdraw). The bird would need the information contained in both the sample and the instructional cue in order to maintain accurate performance. The cue would tell the bird which task was being presented (identity vs. symbolic in Experiment 1; identity vs. delayed simple discrimination in Experiment 2). The sample stimulus would tell the bird which of the two comparison stimuli to process for that task (or whether or not to respond in the delayed simple discrimination). By incorrectly cuing on probe trials, the birds would process a stimulus that was correctly paired with the sample, but would be the wrong task for that trial. Since the comparison stimuli on probe trials would therefore not be consistent with the stimulus being processed, the birds would not have the information necessary for accurate performance. The results obtained in Experiments 1 and 2 are consistent with this prospective account of processing in pigeon DMTS. Prospective processing seemed to be utilized whether the task was a simultaneous choice task (Experiment 1) or a successive task (Experiment 2).

Although the results of these two experiments seem to favor an interpretation based on prospective processing, Honig and Thompson (1982) pointed out that the results are also compatible with an alternative explanation based on retrospective processing. Birds may be responding to samples and cues as compound stimuli, and the decrement seen on probe trials may be due simply to the birds' not knowing what to do on trials with the novel compound stimulus. Maki (personal communication, September 27, 1981) suggested that this problem could be alleviated with a third experiment. He suggested the use of short retention intervals in such a control study to merely demonstrate that accurate performance is possible on probe trials. Such a demonstration would be contrary to the position that poor performance in the present experiments was due to birds' responding to a compound stimulus of the sample and cue, and would favor the prospective processing interpretation given.

\section{REFERENCES}

Grant, D. S. (1981). Stimulus control of information processing in pigeon short-term memory. Learning and Motivation, 12, 19-39.

Honig, W. K., \& Thompson, R. K. R. (1982). Retrospective and prospective processing in animal working memory. In G. $\mathbf{H}$. Bower (Ed.), The psychology of learning and motivation. New York: Academic Press.

Honig, W. K., \& Wasserman, E. A. (1981). Performance of pigeons on delayed simple and conditional discriminations under equivalent training procedures. Learning and Motivation, 12, 149-170.

MAKI, W. S. (1981). Directed forgetting in animals. In N. Spear \& R. Miller (Eds.), Information processing in animals: Memory mechanisms. Hillsdale, NJ: Erlbaum.

MaKI, W. S., \& HegviK, D. K. (1980). Directed forgetting in pigeons. Animal Learning \& Behavior. 8, 567-574.

Maki, W. S., Olson, D., \& REgo, S. (1981). Directed forgetting in pigeons: Analysis of cue functions. Animal Learning \& Behavior, 9, 189-195.

MAKI, W. S., Riley, D. A., \& Leith, C. R. (1976). The role of test stimuli in matching-to-compound samples in pigeons. Animal Learning \& Behavior, 4, 13-21.

Riley, D. A., Cook, R. G., \& LAmb, M. R. (1981). A classification and analysis of short term retention codes in pigeons. In G. H. Bower (Ed.), The psychology of learning and motivation. New York: Academic Press.

Roberts, W. A. (1972). Short-term memory in the pigeon: Effects of repetition and spacing. Journal of the Experimental Psychology, 94, 74-83.

Roberts, W. A., \& Grant, D. S. (1976). Studies of short-term memory in the pigeon using delayed matching to sample. In D. L. Medin, W. A. Roberts, \& R. T. Davis (Eds.), Processes of animal memory. Hillsdale, NJ: Erlbaum.

Roitblat, H. L. (1980). Codes and coding processes in pigeonshort-term memory. Animal Learning \& Behavior, 8, 341-351.

Ruggiero, F. T., \& FlaGg, S. F. (1976). Do animals have memory? In D. L. Medin, W. A. Roberts, \& R. T. Davis (Eds.), Processes of animal memory. Hillsdale, NJ: Elrbaum.

SPEAR, N. (1981). Extending the domain of memory retrieval. In N. E. Spear \& R. R. Miller (Eds.), Information processing in animals: Memory mechanisms. Hillsdale, NJ: Erlbaum.

Stonebraker, T. B., \& Rilling, M. (1981). Control of delayed matching-to-sample performance using directed forgetting techniques. Animal Learning \& Behavior, 9, 196-201.

Stonebraker, T. B., Rilling, M., \& Kendrick, D. F. (1981). Time dependent effects of double cuing in directed forgetting. Animal Learning \& Behavior, 9, 385-394.

Wagner, A. R. (1976). Priming in STM: An information processing mechanism for self generated or retrieval generated depression in performance. In T. J. Tighe \& R. N. Leaton (Eds.), Habituation: Perspectives from child development, animal behavior, and neuropsychology. Hillsdale, NJ: Erlbaum.

Wasserman, E. A. (in press). Prospection and retrospection as processes of animal short-term memory. In D. F. Kendrick, M. Rilling, \& M. R. Denny (Eds.), Animal memory. Hillsdale, NJ: Erlbaum.

(Revision accepted for publication July $16,1984$. 